\title{
Evaluation of the Efficacy of Postoperative Triamcinolone in Endoscopic Dacryocystorhinostomy using Lacrimal Symptom Questionnaire
}

HASAN BURHANETTIN KAPTI ( $\sim$ burhaneddink@hotmail.com )

Ordu University Faculty of Medicine: Ordu Universitesi Tip Fakultesi https://orcid.org/0000-0002-3960$654 X$

HAKAN KORKMAZ

Ordu University Faculty of Medicine: Ordu Universitesi Tip Fakultesi

\section{Research Article}

Keywords: triamcinolone, endoskopic, dacryocystorhinostomy, lacrimal symptom questionnaire.

Posted Date: August 9th, 2021

DOl: https://doi.org/10.21203/rs.3.rs-570610/v1

License: (c) (i) This work is licensed under a Creative Commons Attribution 4.0 International License. Read Full License

Version of Record: A version of this preprint was published at International Ophthalmology on March 31st, 2022. See the published version at https://doi.org/10.1007/s10792-022-02305-6. 


\section{Abstract}

Purpose: To evaluate the effect of postoperative nasal triamcinolone spray use in primary endoscopic dacryocystorhinostomy using The Lacrimal Symptom Questionnaire .

Methods: This study included a series of 50 consecutive retrospective cases with primary endoscopic dacryocystorhinostomy and with silicone stent implantation who were followed up for 6 months postoperatively. Patients using nasal triamcinolone spray for 3 months postoperatively were included in the triamcinolone group. Patients not using triamcinolone were included in the control group. Lacrimal stents were removed 3 months postoperatively. The Lacrimal Symptom Questionnaire (Lac-Q) was performed in the preoperative and postoperative 6th month. Anatomical success, functional success, and changes in lacrimal symptom and social impact scores were compared between the two groups 6 months after surgery.

Results: Results of 48 endoscopic dacryocystorhinostomies performed on 48 patients (23 triamcinolone group, 25 control group) meeting the inclusion criteria were analyzed. The anatomical success rate $(95.7 \%$ vs. $84.0 \%, p=0.350)$ and the functional success rate $(91.3 \%$ vs. $76.0 \%, p=0.249)$ were higher in the triamcinolone group, but this difference was not statistically significant. No complications were observed in the triamcinolone group, whereas complications developed in two patients in the control group $(p=0.490)$. The mean change in total lac-Q score was 11.0 in the triamcinolone group and 9.0 in the control group $(p=0.011)$. The mean change in social impact score was 4.0 in the triamcinolone group and 3.0 in the control group $(p=0.005)$. Mean change in lacrimal symptom score was 6.0 in the triamcinolone group and 6.0 in the control group $(p=0.368)$

Conclusions: Our study indicated that postoperative use of triamcinolone spray increases the success rate of endoscopic dacryocystorhinostomy. also revealed that triamcinolone may also lead to greater improvement in quality of life scores than the control group using a validated questionnaire.

\section{Introduction}

Dacryocystorhinostomy (DCR) is a surgical procedure for distal nasolacrimal duct obstruction. It is defined as creating a functional pathway from the canaliculi to the nose by performing an osteotomy and opening the nasolacrimal sac within the nasal cavity. This technique can be performed by external and endonasal endoscopic approach. Endonasal DCR (EDCR) has advantages such as being less invasive compared to the external approach, causing less hemorrhage, shorter operation time, and preserving the function of orbicularis oculi [1].

The failure rate of EDCR has been reported to be approximately $6 \%-14 \%$. Granuloma formation, abnormal wound healing resulting in fibrosis, and cicatricial closure of the osteotomy site have been stated as causes of failure [2,3]. Steroid treatment inhibits inflammatory response and fibrosis during wound healing. It has been shown that the use of nasal packs impregnated with triamcinolone, a topical corticosteroid, after endoscopic sinus surgery improves surgical results and reduces the risk of 
postoperative complications [4,5] In previous studies, it has been shown that triamcinolone acetonide, a modern topical corticosteroid that binds to high serum proteins, is well tolerated in seasonal and allergic rhinitis and prevents the regrowth of polyps after polyp surgery[6]. There are studies showing the effectiveness of steroids in EDCR. These studies had focused on the anatomical and functional results of steroid use following EDCR surgery and in the postoperative period [7-9].

The Lacrimal Symptom Questionnaire (Lac-Q) is a questionnaire developed to assess symptoms before and after DCR surgery. In the postoperative period, it helps us to measure the improvement in the quality of life by evaluating the social impact as well as lacrimal symptomatology [10]. Its ease of application as well as the reliability and validity of Lac-Q have been demonstrated in studies comparing different surgical techniques in DCR[11,12]. To the best of our knowledge, the present study is the first in which the effect of triamcinolone use after EDCR on quality of life as well as its anatomical and functional results were investigated.

The aim of this study was to anatomically and functionally evaluate the effect of use of intranasal triamcinolone spray on the outcome of surgery after EDCR and to measure its effect on the changes in quality of life using the Lac-Q questionnaire.

\section{Methods}

The medical records of patients who underwent EDCR surgery between January 2018 and December 2020 at Ordu University Faculty of Medicine Training and Research Hospital were retrospectively reviewed. The study was conducted in accordance with the 2013 Helsinki Declaration and was approved by the Ordu University Faculty of Medicine Ethics Committee (2021/116). Informed consent was obtained from each patient

Patients with nasolacrimal duct obstruction over 20 years of age who underwent primary endoscopic DCR surgery and were followed up for at least 6 months were included in this study. /Patients without regular follow-up records, those with unsuccessful DCR history, those with nasal pathology, those who underwent external DCR, and those who had other causes of epiphora such as canalicular obstruction, punctal stenosis, ectropion, and facial paralysis were excluded from the study.

A full ophthalmologic examination was performed on all patients participating in the study. Lacrimal irrigation and probing were performed to evaluate the status of the nasolacrimal passage. An endoscopic examination was performed by an otolaryngologist to exclude nasal cavity lesions. The patients were divided into two groups. Group 1 included patients using intranasal triamcinolone for 3 months postoperatively. Group 2 (control group) included patients who did not use triamcinolone postoperatively.

\section{Surgical technique}

All operations were performed under general anesthesia and by the same surgeon. Adrenaline pad was placed in the nasal mucosa in front of the middle concha to reduce bleeding. An oval mucosal incision 
was made with a scalpel between the middle concha and the inferior concha. Subsequently, the mucosa was removed using an elevator and forceps. Osteotomy was performed using Kerrison forceps. The lacrimal sac was exposed. The lacrimal probe was inserted through the canaliculus and directed to the medial wall of the sac. The medial wall of the sac was stretched with the probe, an incision was made with the help of a scalpel, and the sac was exposed using forceps. The lacrimal probe was viewed endoscopically A silicone tube was passed through the upper and lower canaliculi, and its ends were tied together in the nasal cavity. Postoperatively, all patients received oral antibiotics for one week and betamethasone eye drops four times a day for six weeks. Nasal spray containing triamcinolone was applied once a day to the study group for three months. The patients were postoperatively followed-up in the 1st week, 1st month, 2nd month, 3rd month, and 6th month. Silicone tubes were removed at three months.

\section{Evaluation of Results}

Improvement in lacrimal symptoms was assessed by Lac-Q. Lac-Q questionnaire was administered to all patients by an independent observer before surgery and six months after surgery. As seen in Table 1, the lacrimal symptoms and social impact of the condition were evaluated for each eye. The total Lac-Q score was obtained by summing the lacrimal symptom score and social impact score. In addition, lacrimal irrigation test was performed in all patients who underwent DCR surgery in the 6th postoperative month. Anatomical success was defined as water passing through the lacrimal passage and a patent ostium. Functional success was evaluated by asking the patients whether the epiphora was resolved or not. Recovery of symptoms and complaints was defined as functional success. Moreover, secondary results were determined by observing synechiae, granular tissue formation, and tube-induced inflammation.

\section{Table 1. The Lacrimal Symptom Questionnaire (Lac-Q)}


Which of these five statements is true about the tear duct problem overall in the last 8 weeks?

1. Friends family have commented about the watery eye problem

2. The watery eye problem has caused embarrasment in company

3. The watery/sticky eye problem has interfered with everyday activity like reading and driving

4. The vision is sometimes blurred because of the watery/sticky eye problem

5. Medical attendance: visit to family doctor or eye hospital because of the tear duct problem

Lacrimal symptom score $(\max$ score $=14$ for each eye $)$

For each of the four problems, put a tick in the box next to the statement which best describes the situation over the last eight weeks. Use the left hand column for the left eye and the right hand column for the right eye.
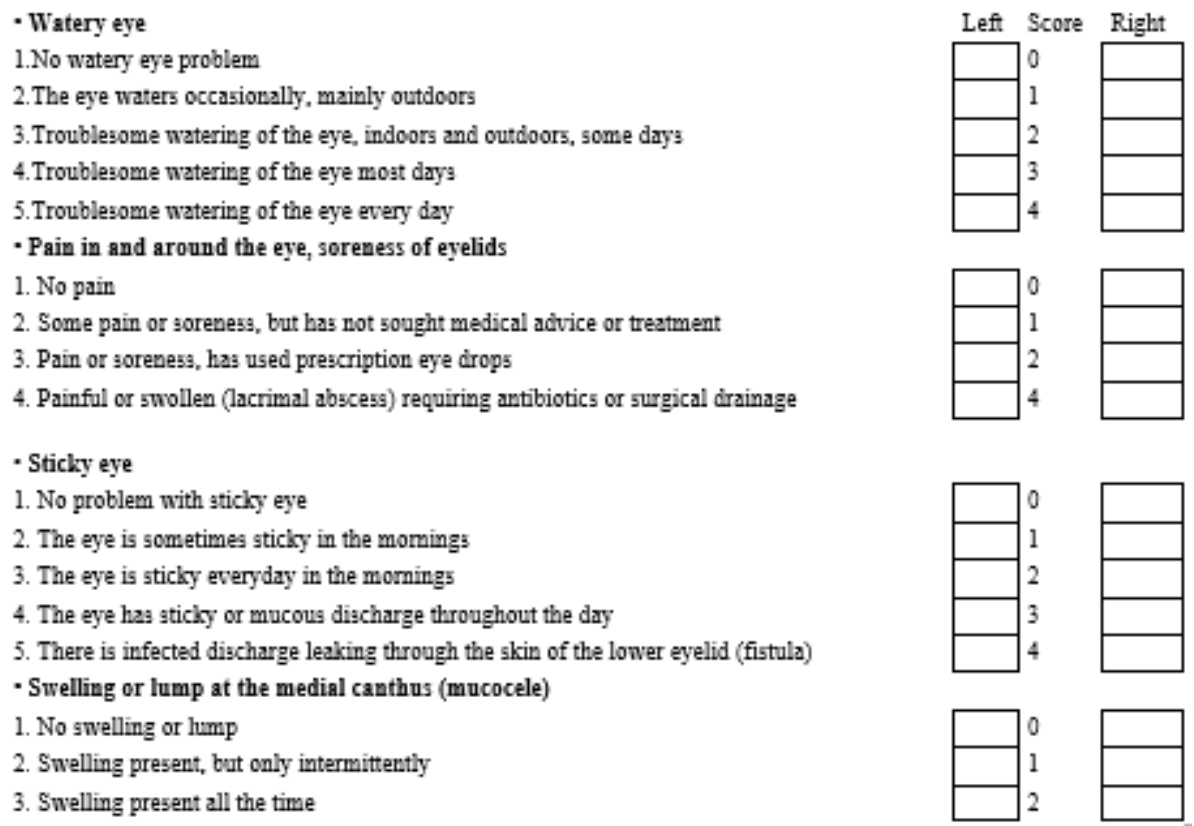

\section{Statistical analysis}

Categorical data were expressed as frequency and percentage, whereas continuous data were expressed as median and interquartile range (IQR). Categorical data were compared using the chi-square or Fisher's exact test, as appropriate. For continuous data, Mann-Whitney U test was used for comparison of independent groups and Wilcoxon test was used for comparison of dependent groups. Data were analyzed with SPSS 20.0 package program. Statistical significance level was accepted as $\mathrm{P}<0.05$.

\section{Results}

During the study period, EDCR was performed in 50 eyes of 50 patients. One patient with canalicular stenosis and one patient who did not attend the 6th month follow-up were excluded from the study. A total of 48 patients, including 20 men (41.5\%) and 28 women (58.5\%), were included in the study. There 
was no significant difference between the two groups in terms of age, laterality, and sex (Table 2). Social impact, lacrimal symptom, and total score analyses were performed with Lac-Q.

Table 2

Descriptive comparison of triamcinolone and control groups

\begin{tabular}{|c|c|c|c|}
\hline & $\begin{array}{l}\text { Triamcinolone } \\
\text { group }(n=23)\end{array}$ & $\begin{array}{l}\text { Control group } \\
(n=25)\end{array}$ & p \\
\hline Age,median (IQR) & $57(40-62)$ & $57(47-63)$ & $0.470^{\mathrm{a}}$ \\
\hline \multicolumn{4}{|l|}{ Sex, n (\%) } \\
\hline Male & $10(43.5 \%)$ & $10(40.0 \%)$ & \multirow[t]{2}{*}{$0.807^{b}$} \\
\hline Female & $13(56.5 \%)$ & $15(60.0 \%)$ & \\
\hline \multicolumn{4}{|l|}{ Laterality, n (\%) } \\
\hline Right & $13(56.5 \%)$ & $13(52.0 \%)$ & \multirow[t]{2}{*}{$0.753^{b}$} \\
\hline Left & $10(43.5 \%)$ & $12(48.0 \%)$ & \\
\hline
\end{tabular}

In the triamcinolone group, the preoperative and postoperative median total Lac-Q scores were 12.0 (IQR, 11.0-13.0) and 1.0 (IQR, 0.0-2.0), respectively. The median change in the total Lac-Q score was 11.0 (IQR, 9.0-12.0). In the control group, the preoperative and postoperative median total Lac-Q scores were 11.0 (IQR, 10.0-12.5) and 1.0 (IQR 1.0-6.5), respectively. The median change in the total Lac-Q score was 9.0 (IQR, 6.5-10.0). The median change in the total Lac-Q score was significantly lower in the control group $(p=0.011)($ Fig. 1).

In the triamcinolone group, the respective preoperative and postoperative median social impact scores were 4.0 (IQR, 4.0-5.0) and 0.0 (IQR, 0.0-0.0). The median change in social impact score was 4.0 (IQR, 3.0-5.0). In the control group, the respective preoperative and postoperative median social impact scores were 4.0 (IQR, 4.0-5.0) and 0.0 (IQR, 0.0-0.2). The median change in social impact score was 3.0 (IQR, 2.5-4.0). The change in the median social impact score between the two groups was statistically significant $(p=0.005)$ (Fig. 2).

In the triamcinolone group, the preoperative and postoperative median lacrimal symptom scores were 7.0 $(\mathrm{IQR}, 6.0-9.0)$ and $1.0(\mathrm{IQR}, 0.0-0.2)$, respectively. The median change in lacrimal symptom score was 6.0 (IQR, 5.0-7.0). In the control group, the respective preoperative and postoperative median lacrimal symptom scores were 8.0 (IQR, 6.5-8.0) and 1.0 (IQR, 0.5-4.0), respectively. The median change in lacrimal symptom score was 6.0 (IQR, 4.5-7.0). The change in median lacrimal symptom score between the two groups was not statistically significant $(p=0.368)($ Fig. 3$)$. 
When anatomical success after EDCR was evaluated, a patent ostium was observed in 22 (95.7\%) of 23 patients in the triamcinolone group and $21(84.0 \%)$ of 25 patients in the control group. When functional results were evaluated, improvement was observed in 21 patients $(91.3 \%)$ in the triamcinolone group and in 19 patients $(76.0 \%)$ in the control group. The rates of anatomical success $(p=0.350)$ and functional success $(p=0.249)$ were higher in the triamcinolone group; however, this difference was not statistically significant. Complications were not observed in the triamcinolone group $(0.0 \%)$, whereas two patients $(8.0 \%)$ developed complications in the control group. Tube-induced inflammation and synechiae developed in one patient each. The number of complications was higher in the control group; however, there was no statistically significant difference between the groups $(p=0.490)($ Table 3$)$.

Table 3

Comparison of anatomical and functional success and complication rates in triamcinolone and control groups

\begin{tabular}{|llll|}
\hline & $\begin{array}{l}\text { Triamcinolone } \\
\text { group }(\mathbf{n = 2 3 )}\end{array}$ & $\begin{array}{l}\text { Control group } \\
(\mathbf{n = 2 5})\end{array}$ & $\mathbf{p}^{\mathbf{a}}$ \\
\hline Anatomical success, $\mathrm{n}(\%)$ & $22(95.7 \%)$ & $21(84.0 \%)$ & 0.350 \\
\hline Functional success, $\mathrm{n}(\%)$ & $21(91.3 \%)$ & $19(76.0 \%)$ & 0.249 \\
\hline Complication, $\mathrm{n}(\%)$ & $0(0.0 \%)$ & $2(8.0 \%)$ & 0.490 \\
\hline a. Fisher's Exact test & & & \\
\hline
\end{tabular}

\section{Discussion}

There are different postsurgical medical treatments to improve the results of DCR. These include topical ocular antibiotics and steroids, topical nasal antibiotics and steroids, nasal vasoconstrictors, and oral antibiotics[13]. However, there is no definitive data demonstrating that these treatments are actually effective. In addition, some studies recommend local antifibrinogenic and anti-inflammatory treatments such as 5-fluorouracil and mitomycin-C[14, 15]. However, a standard postoperative treatment protocol has not been established. On the other hand, although steroids are generally included in postoperative treatment due to their anti-inflammatory effects, there are very few studies on the use of steroids.

Corticosteroids are known to suppress inflammation and fibroblast formation and reduce scarring. Steroids have been shown to have positive effects on wound healing after nasal sinus surgery. It has been demonstrated that the use of oral steroids before and after nasal polypectomy and the use of triamcinolone-impregnated nasal packs after endoscopic sinus surgery can reduce nasal mucosal inflammation [16, 4]. Another study showed that the use of triamcinolone-impregnated nasal packs in endoscopic sinus surgery provides a significant reduction in the incidence of complications such as mucosal edema and crusting [5]. A similar effect was observed in EDCR. There are a few studies evaluating the effect of steroid use in EDCR. Kang et al. found that the functional success rate was significantly higher in the group in which triamcinolone-impregnated nasal pack was used in EDCR 
compared to the control group $(p=0.033)$. The use of triamcinolone was shown to be effective in reducing the incidence of postoperative granulation and epiphora [7]. Ali et al. showed that nasal steroid spray reduced granulation in 43 of 47 patients with granulation after DCR [9]. Further, Jo et al. performed intralesional steroid injection treatment on 65 patients who developed granulation after EDCR and observed regression in all the patients [8]. In the present study, anatomical success $(95.7 \%, 84.0 \%$, respectively) and functional success $(91.3 \%, 76.0 \%$, respectively) were higher in the triamcinolone group compared to the control group. However, the differences were not statistically significant.

There may be many reasons for granulation formation, which is one of the causes of failure after DCR. One of these reasons is believed to be immune response induced by silicone intubation [9]. In the present study, to prevent such a possible reaction, we preferred the use of triamcinolone nasal steroid spray until the 3rd month, when the silicone tubes were removed. Whereas no complications were observed in the triamcinolone group, we observed inflammation due to silicone tube in one patient and intranasal synechiae in another patient in the control group ( $0.0 \%$ and $8.0 \%$, respectively).

While evaluating the success of the surgery after DCR, anatomical and functional successes are generally emphasized. Anatomical success is defined as the presence of a patent ostium, and functional success is defined as the recovery of epiphora $[7,8]$. Another evaluation method focuses on the patient's perception of benefit and improvement of symptoms after surgery. Recently, the use of patient-reported outcome measures has gained importance. The Lac-Q questionnaire, which was developed by Mistry et al., allows the evaluation of lacrimal symptomatology such as epiphora, sticky eye, pain, and swelling, as well as social impact of the disease and the effect on quality of life [10]. The validity and reliability of this method have been demonstrated in several studies. Ali et al. conducted a study on 55 patients who underwent augmented DCR, in which preoperative and postoperative Lac-Q questionnaire survey was performed. The change in the Lac-Q score and anatomical and functional success were analyzed, and the authors found that postoperative scores correlated well with anatomical and functional success rates [11]. In another study comparing external and endoscopic DCR, the Lac-Q questionnaire was applied to 59 patients preoperatively and at the postoperative 4th week, 3rd month, and 6th month. The changes in social impact, lacrimal symptomology, and total scores were compared between the two treatment groups. Preoperative and postoperative differences in total Lac-Q scores, lacrimal symptom scores, and social impact scores were significantly different between endoscopic and external DCR groups $(p=0.008$, $p=0.004$, and $p=0.040$, respectively). In this study, the authors showed that Lac- $Q$ is sensitive enough to highlight the magnitude of the change in lacrimal symptoms and social impact [12]. In the present study, the total Lac-Q score and social impact score were significantly higher in the triamcinolone group, which also showed higher anatomical and functional success compared to the control group $(p=0.011$ and $p=$ 0.005 , respectively). There was no statistically significant difference in the lacrimal symptom score.

The limitations of the present study include the small number of cases, the lack of long-term postoperative follow-up data, and the absence of placebo application in the control group. In addition, we evaluated the anatomical and functional outcomes and the effects of surgery on quality of life at the 
postoperative 6th month. Examination data from the earlier period and the 3rd month when the silicone tubes were removed were not available and could not be shared in the present study.

In conclusion, we demonstrated that the use of topical nasal triamcinolone spray after EDCR is effective in reducing the incidence of postoperative complications. In addition, positive results were obtained in the questionnaires evaluating the social impact and lacrimal symptomatology of the disease. There is a need for prospective, randomized, placebo-controlled studies on this subject with a larger number of patients.

\section{Declarations}

Funding: No funding was received for conducting this study.

Conflicts of interest/Competing interests: The authors have no relevant financial or non-financial interests to disclose

Availability of data and material: The datasets generated during and/or analysed during the current study are available from the corresponding author on reasonable request.

\section{Code availability: Not Applicable}

Authors' contributions: All authors contributed to the study conception and design. All authors read and approved the final manuscript.

Ethics approval: This study was performed in line with the principles of the Declaration of Helsinki. Approval was granted by the Ethics Committee of Ordu University Medical School (Date:2021/No:116).

Consent to participate: Informed consent was obtained from all individual participants included in the study.

Consent for publication: Patients signed informed consent regarding publishing their data and photographs.

\section{References}

1. Harish V, Benger RS (2014) Origins of lacrimal surgery, and evolution of dacryocystorhinostomy to the present. Clin Exp Ophthalmol 42:284-287. https://doi.org/10.1111/ceo.12161

2. Leong SC, Macewen CJ, White PS (2010) A systematic review of outcomes after dacryocystorhinostomy in adults. Am J Rhinol Allergy 24:81-90. https://doi.org/10.2500/ajra.2010.24.3393

3. Keren S, Abergel A, Manor A et al (2020) Endoscopic dacryocystorhinostomy: reasons for failure. Eye (Lond) 34:948-953. https://doi.org/10.1038/s41433-019-0612-y

4. Côté DW, Wright ED (2010) Triamcinolone-impregnated nasal dressing following endoscopic sinus surgery: a randomized, double-blind, placebo-controlled study. Laryngoscope 120:1269-1273. 
https://doi.org/10.1002/lary.20905

5. Sabarinath V, Harish MR, Divakaran S (2017) Triamcinolone Impregnated nasal pack in endoscopic sinus surgery: our experience. Indian J Otolaryngol Head Neck Surg 69:88-92. https://doi.org/10.1007/s12070-016-1041-x

6. Vento SI, Blomgren K, Hytönen M et al (2012) Prevention of relapses of nasal polyposis with intranasal triamcinolone acetonide after polyp surgery: a prospective double-blind, placebocontrolled, randomised study with a 9-month follow-up. Clin Otolaryngol 37:117-123. https://doi.org/10.1111/j.1749-4486.2012.02455.x

7. Kang TS, Won YK, Kim JY et al (2021) Efficacy of triamcinolone-soaked nasal packing on endoscopic dacryocystorhinostomy. Ophthalmic Plast Reconstr Surg 37:44-47. https://doi.org/10.1097/iop.0000000000001791

8. Jo A, Lee SH, Song WC, Shin HJ (2018) Effects of ostium granulomas and intralesional steroid injections on the surgical outcome in endoscopic dacryocystorhinostomy. Graefes Arch Clin Exp Ophthalmol 256:1993-2000. https://doi.org/10.1007/s00417-018-4024-7

9. Ali MJ, Wormald PJ, Psaltis AJ (2015) The dacryocystorhinostomy ostium granulomas: classification, Indications for treatment, management modalities and outcomes. Orbit 34:146-151. https://doi.org/10.3109/01676830.2015.1014510

10. Mistry N, Rockley TJ, Reynolds T, Hopkins C (2011) Development and validation of a symptom questionnaire for recording outcomes in adult lacrimal surgery. Rhinology 49:538-545. https://doi.org/10.4193/rhino11.042

11. Ali MJ, Iram S, Ali MH, Naik MN (2017) Assessing the Outcomes of Powered Endoscopic Dacryocystorhinostomy in Adults Using the Lacrimal Symptom (Lac-Q) Questionnaire. Ophthalmic Plast Reconstr Surg 33:65-68. https://doi.org/10.1097/iop.0000000000000660

12. Wong WK, Dean S, Nair S (2018) Comparison between endoscopic and external dacryocystorhinostomy by using the Lacrimal Symptom Questionnaire: A pilot study. Am J Rhinol Allergy 32:46-51. https://doi.org/10.2500/ajra.2018.32.4494

13. Vinciguerra A, Nonis A, Resti AG et al (2020) Impact of post-surgical therapies on endoscopic and external dacryocystorhinostomy: systematic review and meta-analysis. Am J Rhinol Allergy 34:846856. https://doi.org/10.1177/1945892420945218

14. Phelps PO, Abariga SA, Cowling BJ et al (2020) Antimetabolites as an adjunct to dacryocystorhinostomy for nasolacrimal duct obstruction. Cochrane Database Syst Rev 4:CD012309. https://doi.org/10.1002/14651858.cd012309.pub2

15. Do JR, Lee H, Baek S et al (2016) Efficacy of postoperative mitomycin-C eye drops on the clinical outcome in endoscopic dacryocystorhinostomy. Graefes Arch Clin Exp Ophthalmol 254:785-790. https://doi.org/10.1007/s00417-015-3229-2

16. Wright ED, Agrawal S (2007) Impact of perioperative systemic steroids on surgical outcomes in patients with chronic rhinosinusitis with polyposis: evaluation with the novel Perioperative Sinus 
Endoscopy (POSE) scoring system. Laryngoscope 117:1-28.

https://doi.org/10.1097/mlg.0b013e31814842f8

Figures

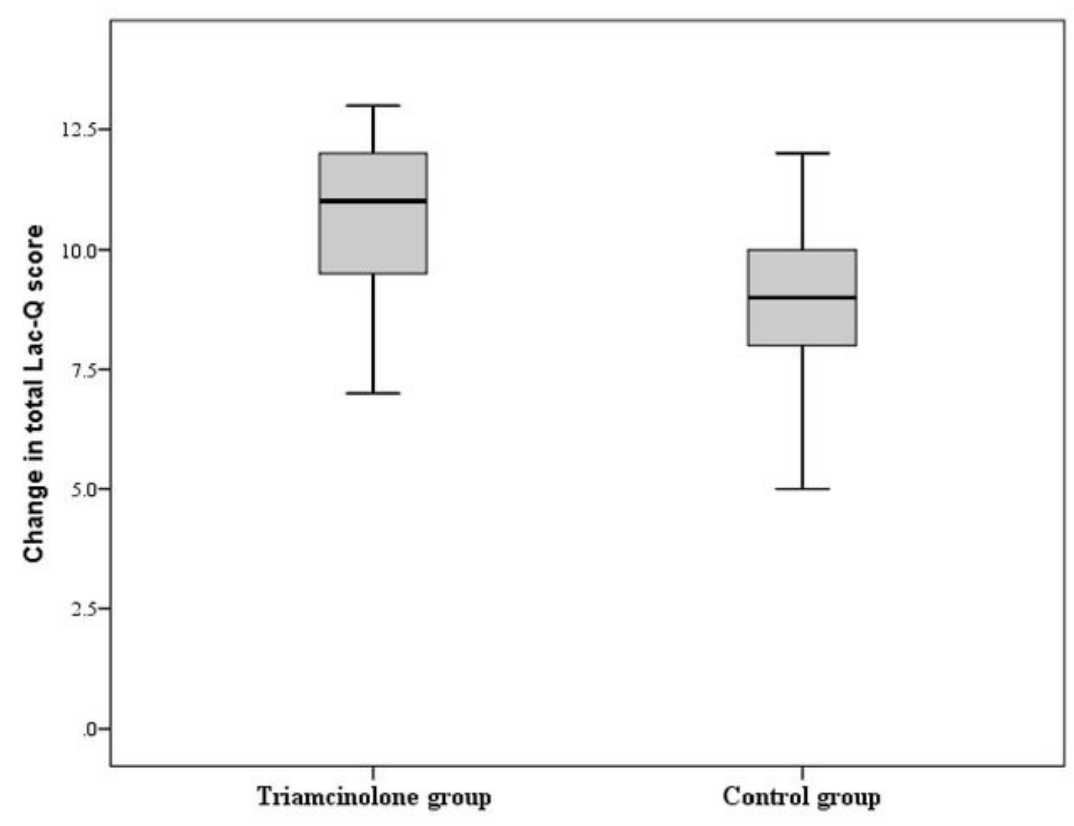

Figure 1

Page $11 / 13$ 
Box plot, depicting the change in total Lacrimal Symptom Questionnaire (Lac-Q) scores of patients using triamcinolone and not using triamcinolone after EDCR

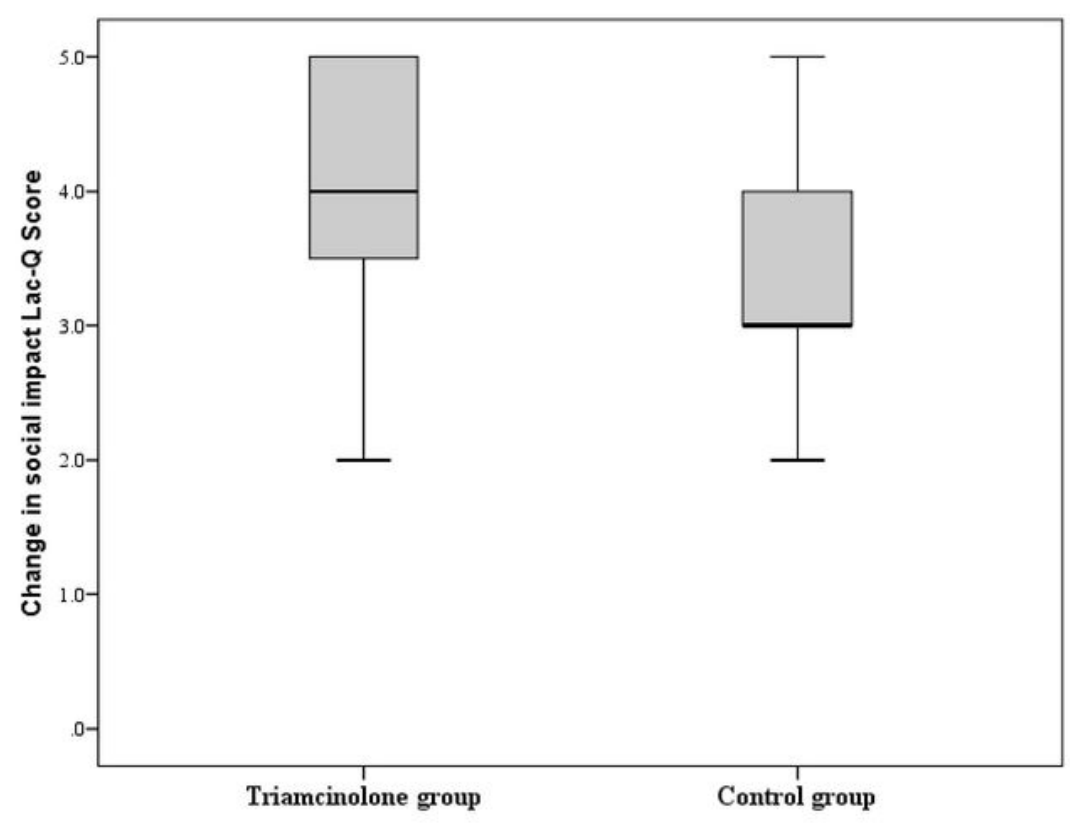

Figure 2

Box plot, depicting the change in social impact scores of patients using triamcinolone and not using triamcinolone after EDCR 


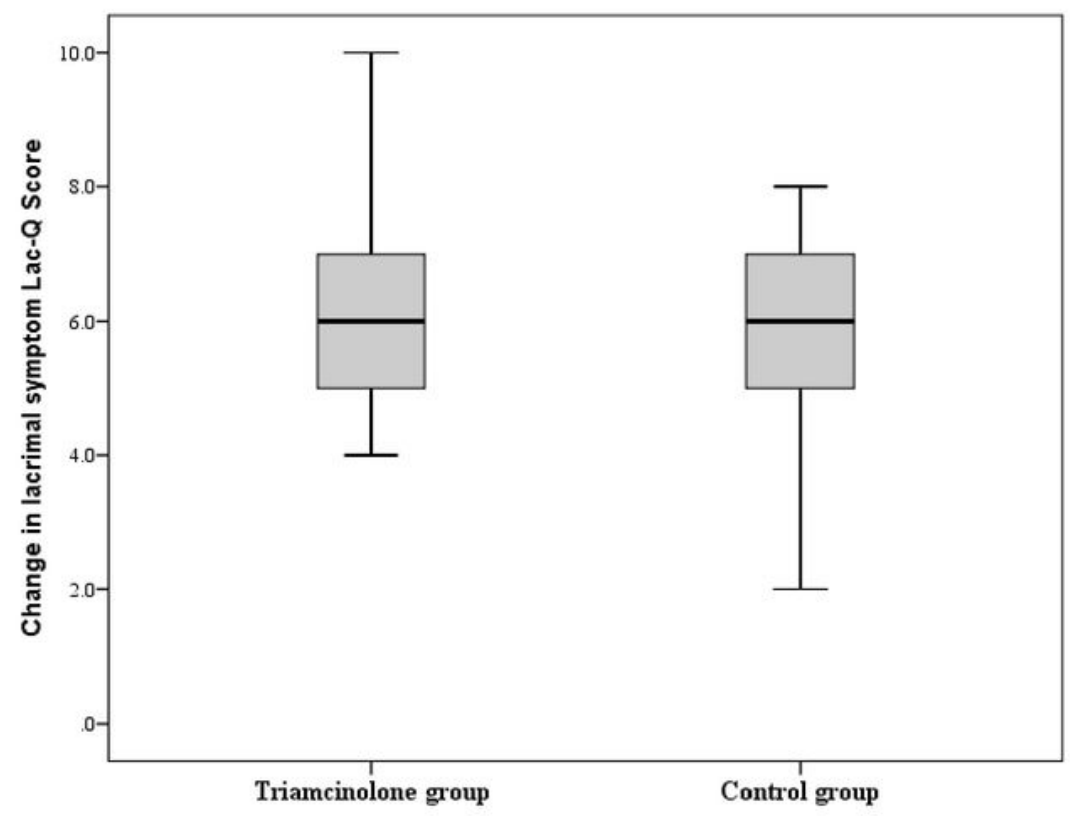

Figure 3

Box plot, depicting the change in lacrimal symptom scores of patients using triamcinolone and not using triamcinolone after EDCR 